\title{
COMETARY EMISSION SPECTRA IN THE VISUAL REGION
}

\author{
P. Swings, A. McKellar, and R. Minkowski \\ McDonald, Dominion Astrophysical, and Mount Wilson Observatories \\ Received May 10, 1943

\section{ABSTRACT}

A list of wave lengths of the emission features occurring in the region $\lambda>4800$ has been compiled partly on the basis of previously published data but mainly from measurements of spectrograms of the recent bright comets $1940 \mathrm{c}$ and $1942 \mathrm{~g}$ obtained at the McDonald, the Mount Wilson, and the Dominion Astrophysical observatories. It is indicated that certain of the more prominent features behave, with respect to the comet's heliocentric distance, as emissions from polyatomic molecules would be expected to do. Comparison of the cometary spectrum with that of an oxyammonia flame leads to the suggested identification of a number of the emission features, among them the strong " $\lambda 6300$ group," as due to a dissociation product of ammonia, probably $\mathrm{NH}_{2}$.

\section{INTRODUCTION}

Relatively few observations of the spectra of comets in the visual region $(\lambda>5500)$ have been made up to the present time. Hence the yellow and near-red regions are comparatively little known. The reasons for this doubtless include the facts that until recently panchromatic plates have been much less sensitive than ordinary blue-sensitive plates, that only very recently have fast enough forms of grating spectrographs been available to enable spectrograms of moderate dispersion of such faint and diffuse objects as cometary heads to be secured, and that no really bright comets have appeared during the past few decades. The only emission bands in this spectral region for which unquestionable identifications have been given are those of the Swan system of $C_{2}$. Also, of course, for several comets which have approached the sun very closely, the sodium $D$-lines have occurred strongly in emission, and possibly a few other atomic lines have been noted.

Recently a considerable number of plates of Comet Cunningham (1940c) and Comet Whipple II (1942g), covering the visual spectral region, have been obtained, using the large reflectors at the McDonald, the Mount Wilson, and the Dominion Astrophysical observatories. It would therefore now seem desirable and profitable to present the results of measurements on these plates, including the limited material available from previous studies, and to assemble and discuss all the wave-length data on cometary spectra for the region $\lambda>4800$.

\section{THE WAVE-IENGTH DATA}

The wave lengths of the emission features to the red of $\lambda 4800$, from which the wellidentified $C_{2}$ and $C N$ bands have been excluded, ${ }^{1}$ are given in three tables. Table 1 presents the results of measurements on Mount Wilson and on McDonald Observatory spectrograms and includes, as well, the results of the earlier work of other investigators on Comet Brooks (1911) and of measurements on Mount Wilson spectrograms of Comet Peltier (1936a) and Comet Finsler (1937f). Table 2 gives the detailed results of the measurements on the Victoria spectrograms.

The McDonald Observatory measurements were made on seven plates of Comet 1940c and two plates of Comet 1942g. The plates of Comet 1940c were taken with a glass spectrograph giving a dispersion of $260 \mathrm{~A} / \mathrm{mm}$ at $\lambda$ 5000; those of Comet 1942g have a

1 The two bands, $\lambda 6053$ and $\lambda 6121$, of the $\Delta v=-2$ sequence of the $C_{2}$ Swan system have been included because they are probably blended with complex groups. All the Swan system transitions from 0,2 to 5,7 were observed in the spectrum of Comet Cunningham. 


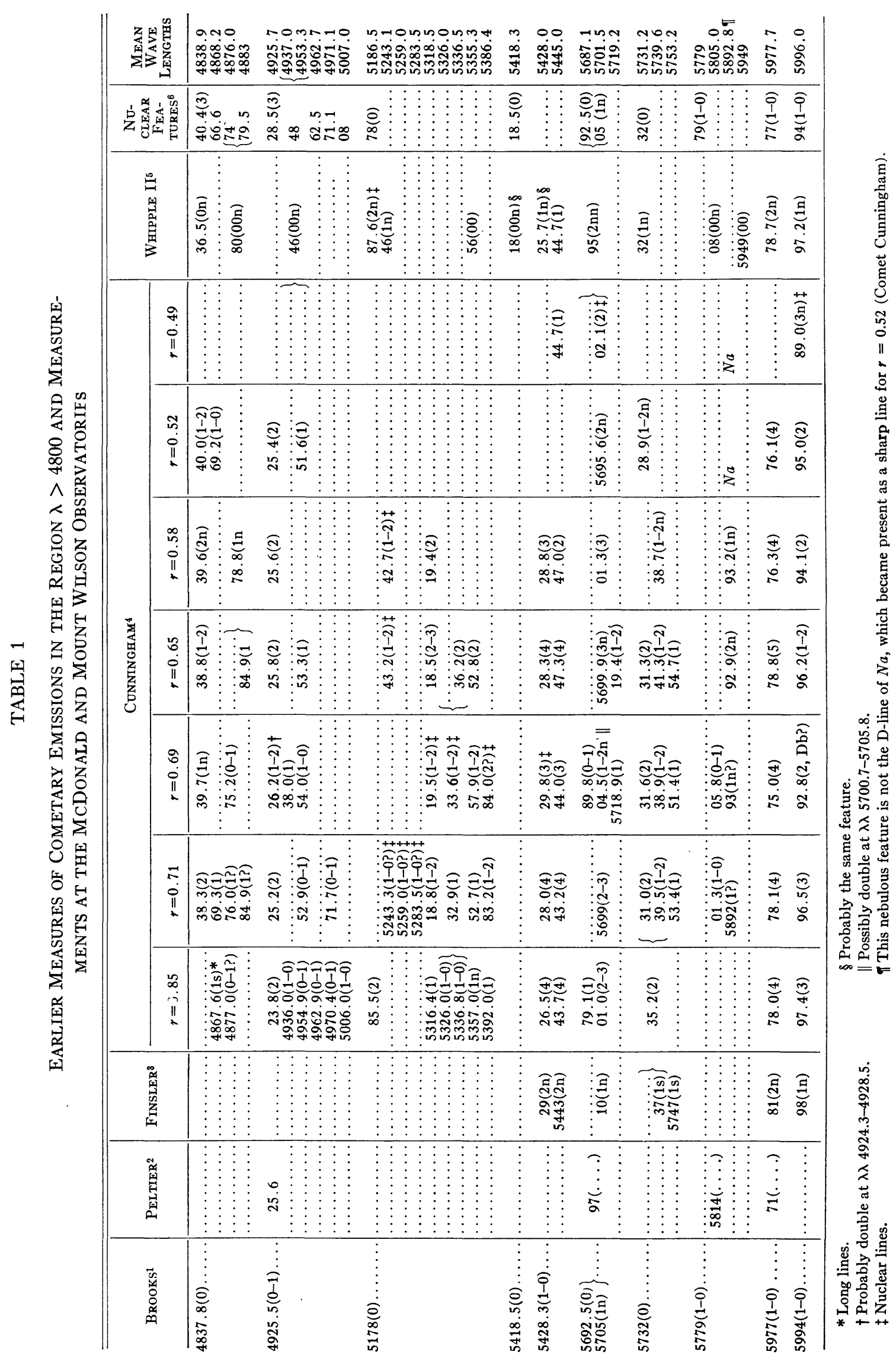




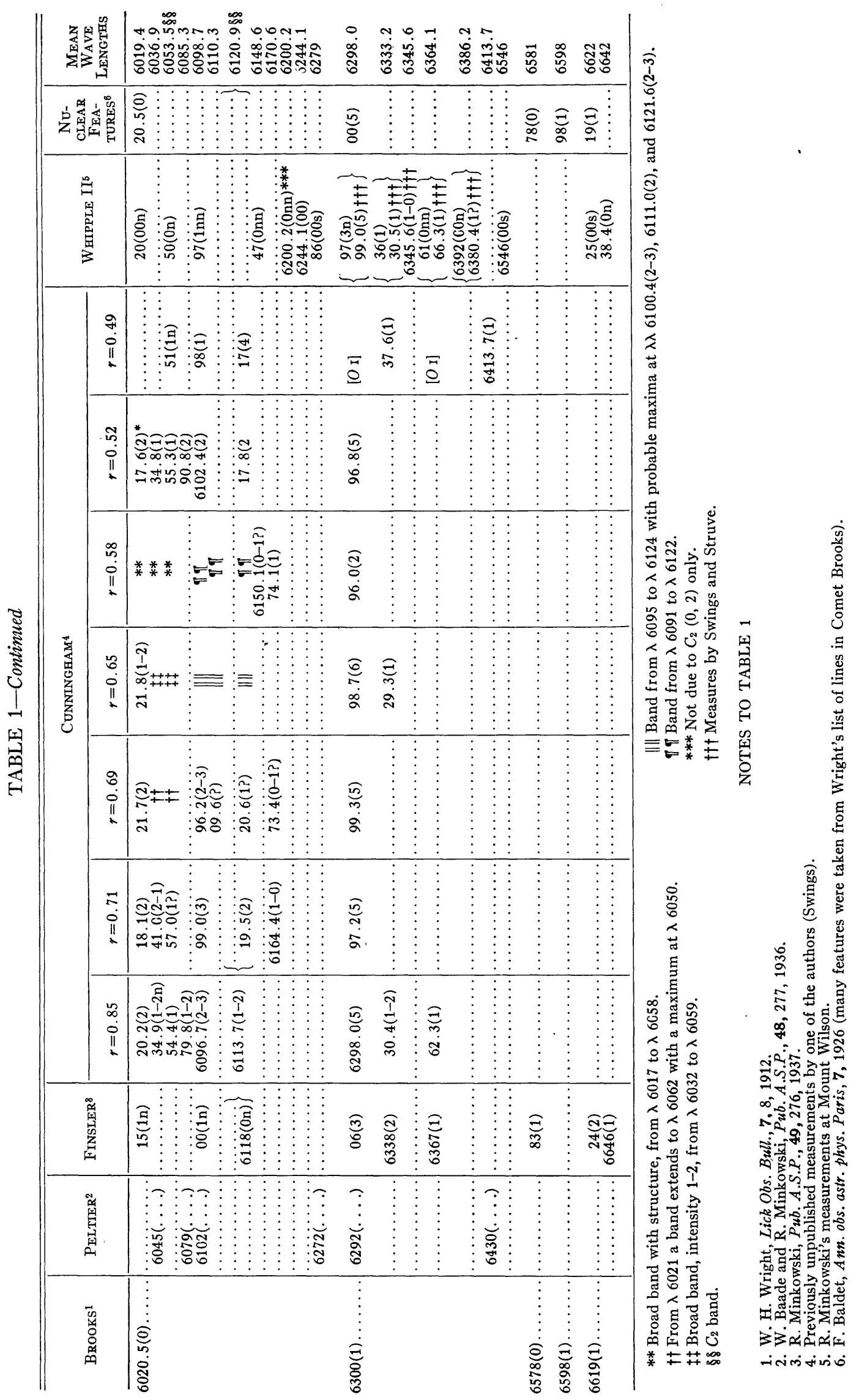


dispersion of $300 \mathrm{~A} / \mathrm{mm}$ at $\lambda 6300$. The Victoria plates were all obtained with the singleprism form of the spectrograph and, except the first of the two of Comet 1942g at $r=1.39$, with a camera giving a dispersion of approximately $225 \mathrm{~A} / \mathrm{mm}$ at $H a$. This one exception was secured with the lower dispersion of $400 \mathrm{~A} / \mathrm{mm}$ at $H a$. For Comet

TABLE $2^{*}$

Victoria Measurements of Emission Bands in Visual Region of Cometary Spectra

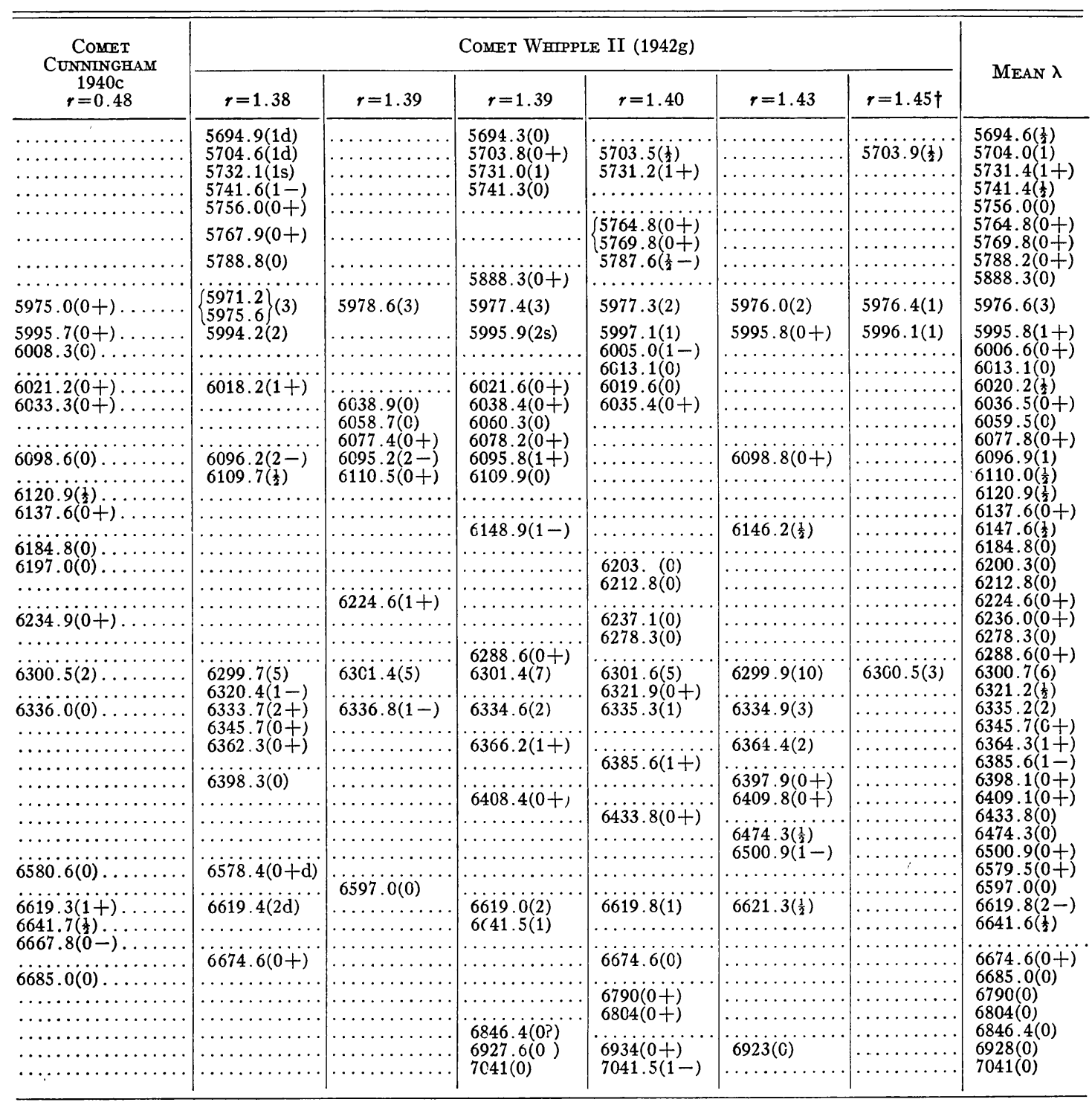

* Excluding, as noted in the text, the well-identified $C_{2}$ and $C N$ bands.

$\dagger$ Weak plate.

1940c Agfa Superpan Press emulsion was used, while for Comet 1942g the Victoria spectra were obtained on Eastman 103-F plates. The Mount Wilson material for Comet $1942 \mathrm{~g}$ was based on two spectrograms obtained on Eastman 103-F emulsion with a grating spectrograph giving a dispersion of $400 \mathrm{~A} / \mathrm{mm}$.

The absolute accuracy of the wave lengths may be estimated from an examination of both the internal consistency of the measurements on the various spectrograms from 
TABLE 3

Final Mean WAVE Lengths OF COMETARY EMissions $\lambda>4800$ and THEIR COMPARISON WITH THE EMISSION SPECTRUM OF THE OXYAMMONIA FLAME

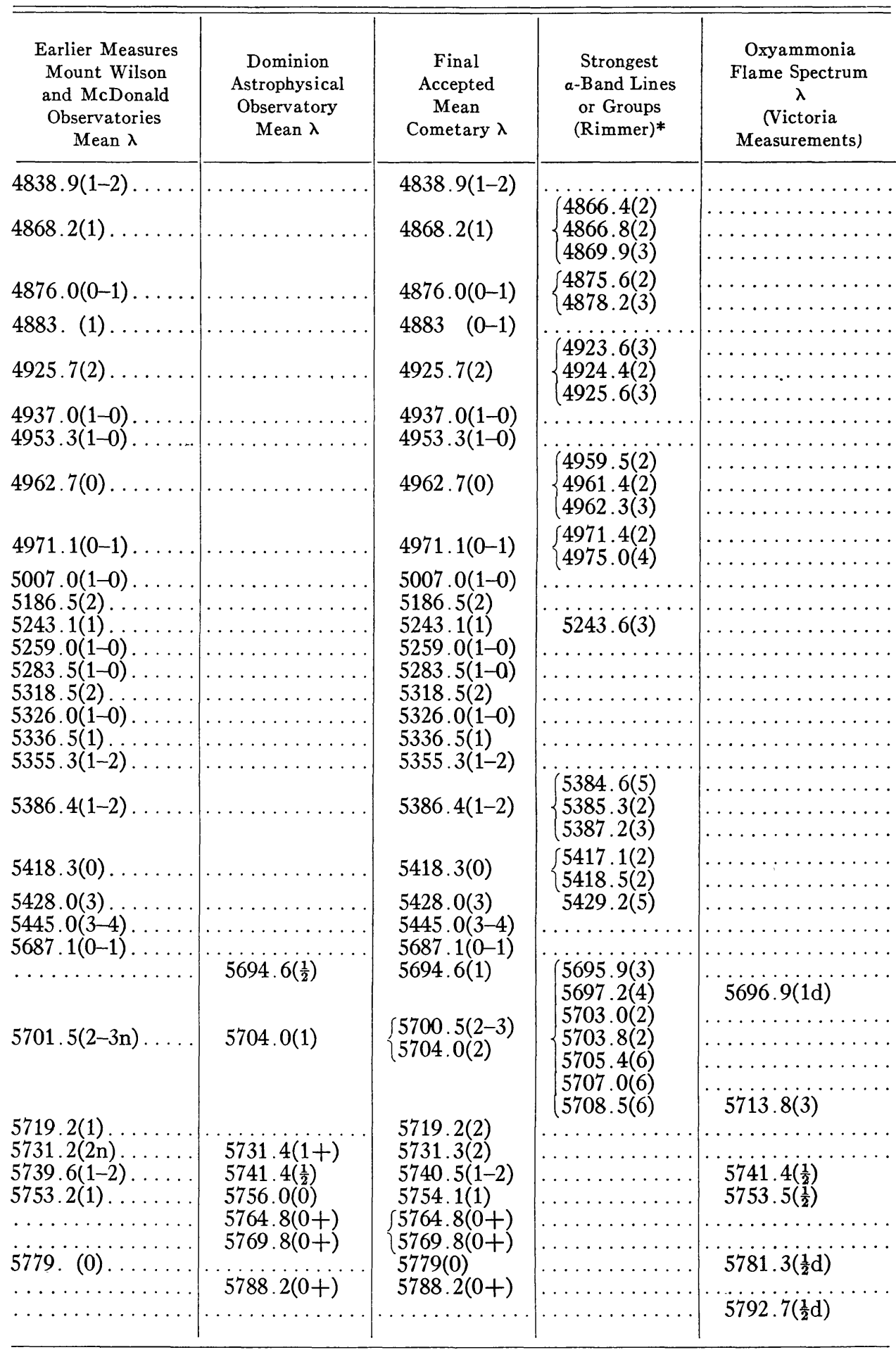

* Proc. R. Soc., A, 103, 696, 1923. 
COMET SPECTRA

TABLE 3-Continued

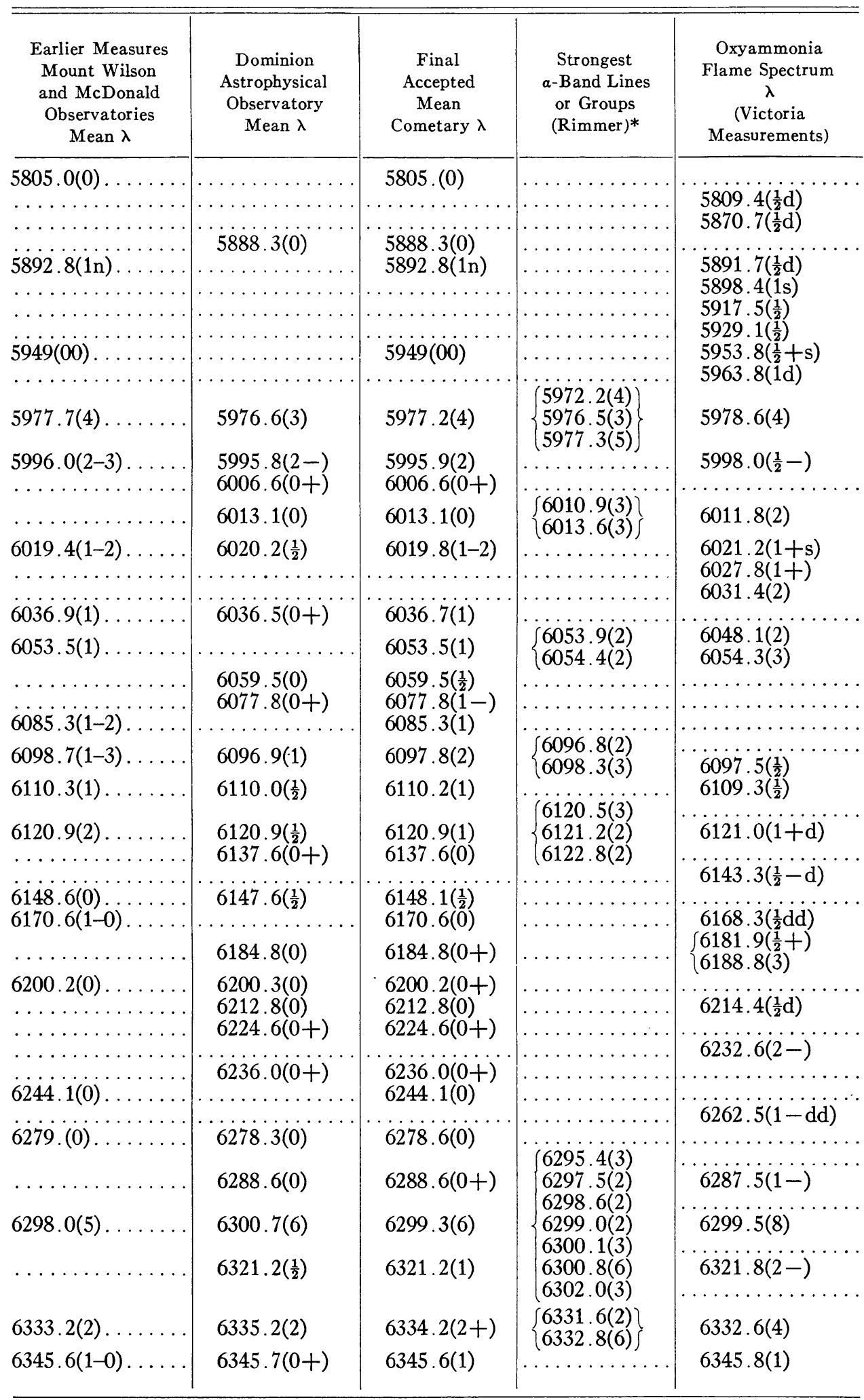


TABLE 3-Continued

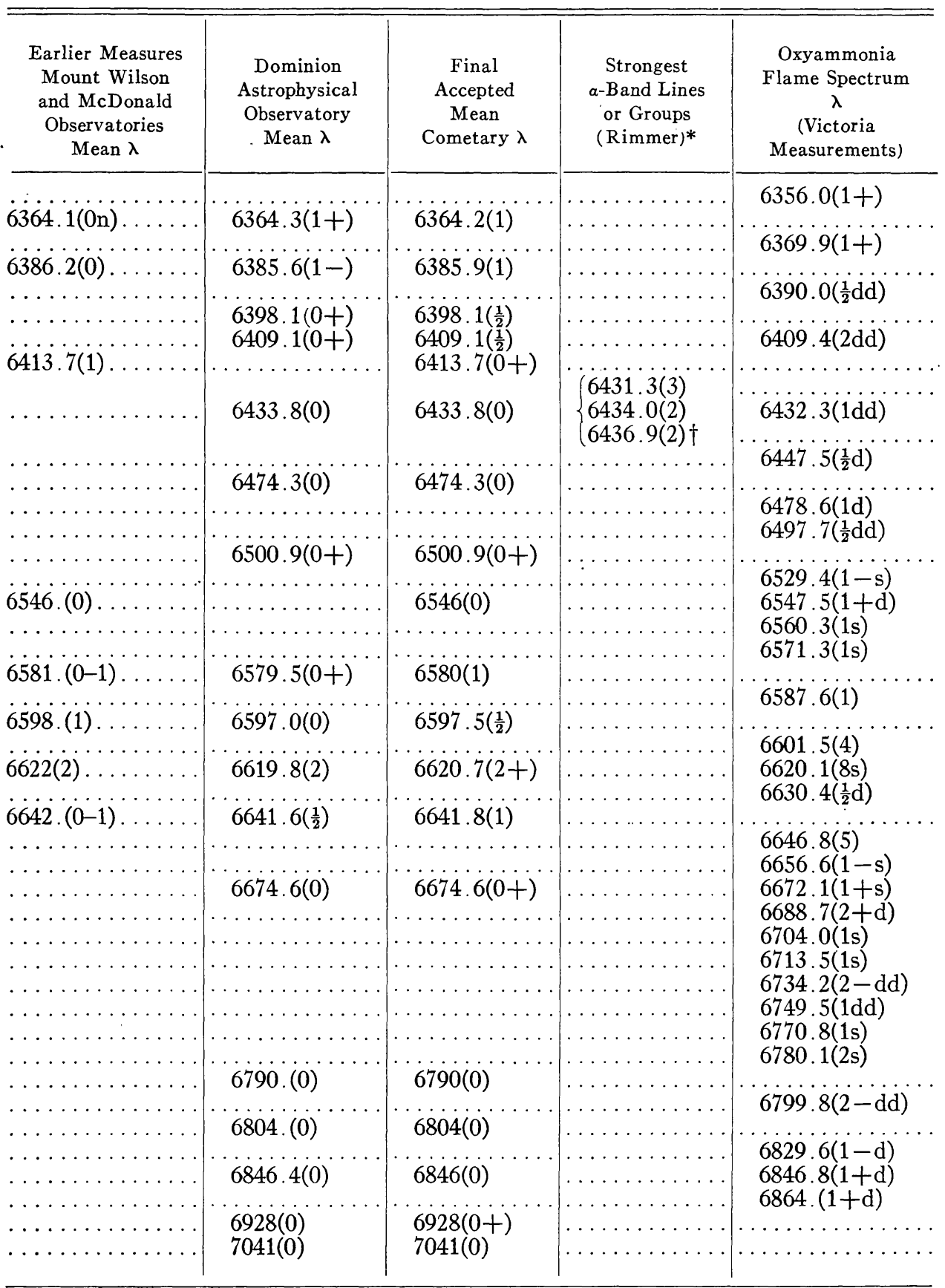

$\dagger$ No measurements beyond $\lambda 6437$.

one observatory and the agreement of the results from the three institutions. From such examinations it would appear that the final mean accepted wave lengths given in Table 3 should be accurate to within a few tenths of an angstrom, especially for the stronger features. It should be noted that most of the emissions, although almost certainly molecular bands, appear fairly narrow and sharp, similar to atomic lines, and, unless it is otherwise definitely indicated, the wave lengths correspond to their intensity maxima. It should also be remarked that a few of the faintest features listed, especially those 
which are concentrated near the cometary nucleus where the reflected solar spectrum is strong, may possibly be spurious, although it is not thought that many are.

\section{DISCUSSION}

The strongest unidentified emission feature in the region of cometary spectra under discussion is the one at $\lambda 6300$. Its total width (on the Victoria plates) is from about $\lambda 6296$ to $\lambda 6304$. With respect to heliocentric distance, it appears to behave in a manner similar to that of the $\lambda 4050$ group of cometary bands, recently shown by Herzberg ${ }^{2}$ to be due to the triatomic molecule $C H_{2}$. At large heliocentric distances, $\lambda 6300$ stands out conspicuously; for example, in the spectrum of Comet $1942 \mathrm{~g}$ at $r=1.40$ astronomical units, there is hardly any trace of $C H$, and the $N H$ and $C_{2}$ bands are weak, but $\lambda 6300$ and the $\lambda 4050 \mathrm{CH}_{2}$ group are both quite strong. A number of features near $\lambda 6300$, particularly one at $\lambda 6334$, apparently behave similarly with respect to heliocentric distance. This group of emissions, which we shall refer to as the " $\lambda 6300$ group," since it resembles in general behavior the bands near $\lambda 4050$, which up to the present time constitute the only cometary emission features known to be due to a polyatomic molecule, may very likely itself be due to a molecule made up of more than two atoms. It appears that the behavior of several other of the stronger cometary emissions, including $\lambda 6620$ and $\lambda$ 5976, may be similar to that of the $\lambda 6300$ group.

One identification for the $\lambda 6300$ group suggests itself, since the strongest feature appearing in the visual region of the spectrum of the oxyammonia flame occurs at $\lambda 6300$. In the flame spectrum this is related to the so-called alpha band of ammonia which covers a large part of the yellow and near-red spectral regions and which has been photographed in the spectra of many different flame ${ }^{3}$ and discharge-tube ${ }^{4}$ sources. Its structure, as revealed by the study of Rimmer, who used relatively high dispersion, is very complex. $\mathrm{He}$ measured some three hundred individual lines of the more than an estimated three thousand observable lines. Reproductions of low-dispersion spectrograms of the hydrogen-nitrous oxide and methane-nitrous oxide flames, in which the alpha band is present, have recently been published by Gaydon. ${ }^{3}$ In the hydrogen-nitrous oxide flame spectrum two intensity maxima at about $\lambda 6300$ and $\lambda$ 6330, which Gaydon found to vary somewhat in intensity from plate to plate, are outstanding; but for the methane-nitrous oxide flame these features are not at all prominent. From his observations Gaydon concluded that "it may be that the structures $[\lambda 6300$ and $\lambda$ 6330] are not of the same origin as the rest of the alpha band or that for some reason they are particularly sensitive to changes in the temperature of the flame." In Eder and Valenta's ${ }^{3}$ reproduction of the spectrum of the ammonia-oxygen flame the features at $\lambda 6300$ and $\lambda 6330$ are conspicuous. In 1927 Hulthén and Nakamura ${ }^{5}$ suggested that the alpha band "is emitted by the excited $\mathrm{NH}_{3}$ or $\mathrm{NH}_{2}$ molecules or a mixture of both, as one can distinguish with some degree of certainty two different systems in alpha." Whatever doubt may remain as to the molecules which actually give rise to the alpha band, it is almost certain that they must be a polyatomic nitrogen-hydrogen combination or combinations (likely not ionized), and it is almost certain that $\mathrm{NH}_{2}$ plays the dominant role.

The electronic structure of $\mathrm{NH}_{2}$ has been considered by Mulliken, ${ }^{6}$ and he has kindly provided us with some information additional to that given in the reference cited. The energy-level diagram (Fig. 1) indicates the nature of the expected transitions on the

${ }^{2}$ Rev. Mod. Phys., 14, 195, 1942; Ap. J., 96, 314, 1942.

${ }^{3}$ Eder, Denkschr. Wien. Akad., 60, 1, 1893, Eder and Valenta, Atlas typischer Spektren (Vienna), p. 10, 1911; Reis, Zs.f. phys. Chem., 76, 560, 1911; Fowler and Badami (hydrogen-nitrous oxide flame), Proc. $R$. Soc., $A, 133,325,1931$; Gaydon (hydrogen-nitrous oxide and methane-nitrous oxide flames), Proc. R. Soc., $A, 181,197,1942$.

${ }^{4}$ Rimmer, Proc. R. Soc., A, 103, 696, 1923.

${ }^{5}$ Nature, 119, 235, $1927 . \quad{ }^{6}$ J. Chem. Phys., 1, 500, 1933 (Table 1). 
assumption that the apex angle is fairly wide, as may be inferred by analogy with Herzberg's interpretation of the $\lambda 4050$ cometary group of $\mathrm{CH}_{2}$ bands. $^{2}$ The diagram suggests that there might be observable $\mathrm{NH}_{2}$ spectra in the visible region, for it seems hardly likely that the interval between ${ }^{2} \mathrm{~B}_{1}$ and ${ }^{2} \mathrm{~B}_{2}$ could be more than 4 volts. The position of the level ${ }^{2} \mathrm{~A}_{1}$ would depend a good deal upon the apex angle; for a smaller apex angle such as that of $\mathrm{H}_{2} \mathrm{O}$ the levels ${ }^{2} \mathrm{~A}_{1}$ and ${ }^{2} \mathrm{~B}_{2}$ might be close together or might even reverse in position.

From these considerations it appears that there could be either one or two moderately intense electronic band systems of $\mathrm{NH}_{2}$ in the visible region. It is also possible that the molecule could have an almost metastable level in case the highest level shown in Figure 1 should actually be the middle one. However, this is improbable. ${ }^{7}$

Certain general considerations which are in harmony with the proposed presence of $\mathrm{NH}_{2}$ in cometary heads may be pointed out. The $\mathrm{NH}$ bands occur strongly in cometary spectra. The presence of $N H$ molecules is most reasonably accounted for as a result

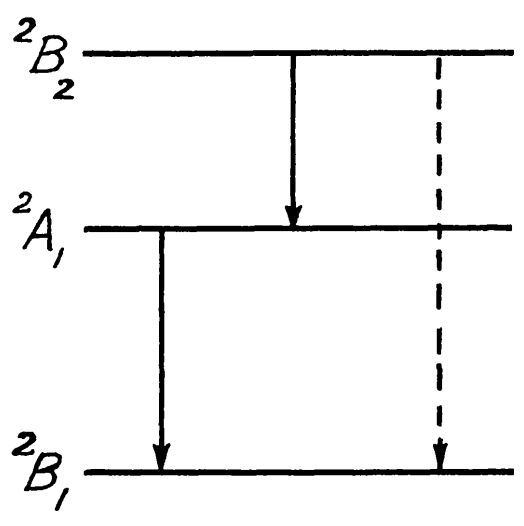

FIG. 1.-Electronic energy-level diagram of $\mathrm{NH}_{2}$. of the photodissociation of chemically stable molecules, probably ammonia, $\mathrm{NH}_{3}$, liberated from the solid constituents of the cometary nucleus. Since it is $\mathrm{known}^{8}$ that the photo-chemical decomposition of ammonia takes place almost exclusively according to the reaction

$$
\mathrm{NH}_{3}+h \nu \rightarrow \mathrm{NH}_{2}+\mathrm{H}
$$

the presence in cometary heads of $\mathrm{NH}_{2}$ is most probable. Data from which it may be inferred that the somewhat analogous dissociation process, with carbon replacing nitrogen, is taking place in cometary gases are very definite. In this case the parentmolecule is probably $\mathrm{CH}_{4}$ (and perhaps also other saturated hydrocarbons), and the $C H$ as well as the $\mathrm{CH}_{2}$ bands are known to occur in cometary spectra.

In the absence of an analysis of the alpha band, it is impossible to predict in detail the structure of the emission spectrum expected from cometary $\mathrm{NH}_{2}$ molecules excited by the resonance mechanism, taking into consideration the profile of the exciting solar radiation. All we can do is to compare as well as possible the wave lengths of the emission maxima in the spectrum of a source of the alpha bands with the emission features in the cometary spectrum. It must be understood that, since we cannot duplicate in the production of the alpha band in the laboratory the excitation conditions obtaining in a cometary head (generally speaking, this corresponds to an effective temperature of a few hundred degrees absolute), slight differences in wave length and greater ones in intensity would be expected in any comparison made.

In order to try to obtain as valid a comparison as possible, an oxyammonia flame was set up in the laboratory at the Dominion Astrophysical Observatory. The ammonia was obtained by heating concentrated ammonium hydroxide and the oxygen from a small commercial tank; the gas was burned, using a glass-blowing torch. The ammonia (undried) came through the central orifice of the burner and the oxygen through a circular opening surrounding the orifice. There was thus no mixing of the gases before combustion; the result attained was therefore very closely that of ammonia burning in an

${ }^{7}$ The data on the electronic structure of $\mathrm{NH}_{2}$ are quoted from a private communication from Dr. R. S. Mulliken.

${ }^{8}$ Farkas and Harteck, Zs. $f$. phys. Chem., 27B, 111, 1934. We do not exclude the possibility that hydrazine molecules $\left(\mathrm{N}_{2} \mathrm{H}_{4}\right)$ might play some role in the production of $\mathrm{NH}_{2}$ or $\mathrm{NH}$. 


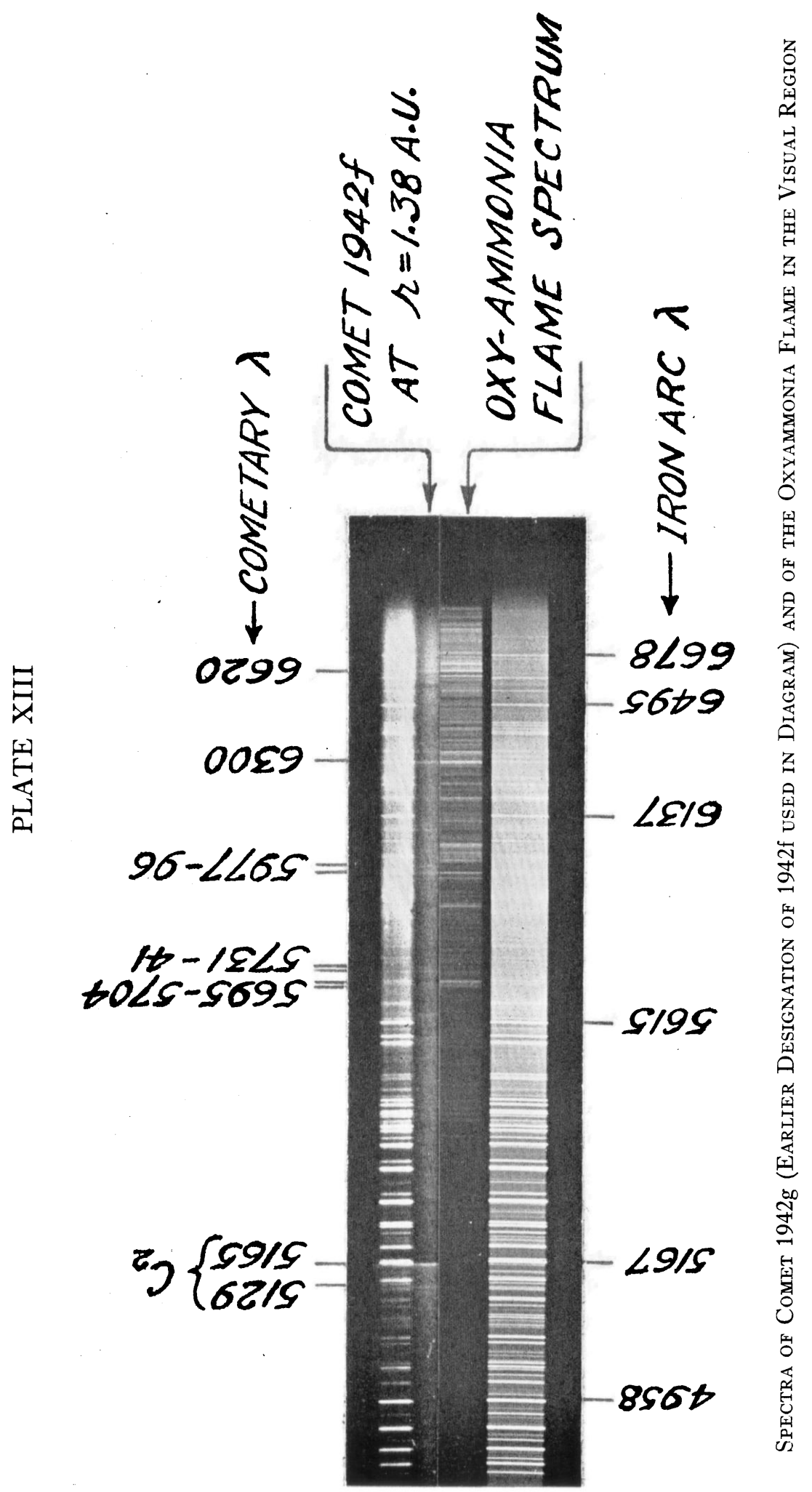


atmosphere of oxygen. Spectrograms of the yellow-colored flame were obtained with a stellar spectrograph (single-glass prism), giving a dispersion of $130 \mathrm{~A} / \mathrm{mm}$ at $\mathrm{Ha}$ (almost twice that used for the comets). The same type of plate used for Comet 1942g (Eastman 103-F) was employed. A reproduction of enlargements of a spectrogram of Comet 1942g and of the oxyammonia flame spectrum obtained at Victoria are shown side by side in Plate XIII. In these reproductions the prominent features at $\lambda 6300$ and $\lambda 6333$ match well, as also do a number of others, notably those at $\lambda 5977$ and $\lambda 6620$. The wave lengths of all the emission features on two of the oxyammonia flame spectrograms were measured. They are listed in Table 3, which, as well as presenting the finally adopted mean wave lengths of the cometary emissions, shows a comparison between these and the wave lengths of the emission maxima in the oxyammonia flame spectrum. The table also includes a column giving wave lengths of strong lines and groups of lines measured by Rimmer on high-dispersion spectrograms of the alpha band. It is seen that there are some twenty approximate wave-length coincidences (taking about 2 angstrom units as the maximum allowable difference) between the forty-odd cometary wave lengths and sixty-seven flame-spectrum wave lengths. The general correspondence in relative intensity of these coincidences also seems fairly good. It may be noted that the only features, other than those listed here, obtained on plates of the oxyammonia flame spectrum using Eastman F-type emulsion and a quartz spectrograph, were the $\lambda 3360 \cdot N H$ band and the $\lambda 3064$ and $\lambda 2811 O H$ bands.

We suggest that a number of these coincidences are real and that several of the strongest features in the emission spectra of the comets are due to the molecule responsible for at least part of the oxyammonia flame spectrum, this molecule probably being $\mathrm{NH}_{2}$. This identification must be considered to be somewhat tentative. For example, the intense emissions at $\lambda \lambda$ 6299.3, 5977.2, 5694.6, and 5445.0 (and perhaps the weaker $\lambda$ 6674.6) are so spaced in wave length as to be interpretable as a $v^{\prime}$ progression of bands emitted by a diatomic molecule. However, the prominence of the strong $\lambda \lambda(6299.3$, 5977.2 , etc., bands at comparatively large distances from the sun, as stressed earlier, and the wave-length agreements with the oxyammonia flame spectrum definitely favor the polyatomic emitter.

Assuming this tentative identification to be true, there remains, however, a considerable number of unidentified features. Concerning these the following remarks may be made:

a) The cometary line, $\lambda 4839$, is near to the strong Vegard-Kaplan band of $N_{2}$ at $\lambda$ 4837.1. However, this identification is very doubtful indeed, since other Vegard-Kaplan bands of similar intensity in the spectrum of the night sky are absent.

b) The features at $\lambda 4868$ and $\lambda 4971$ are close to the wave lengths of the unidentified absorption bands at $\lambda 4866$ and $\lambda 4975$ first found by Sanford ${ }^{9}$ and Shane ${ }^{10}$ in the spectra of certain N-type stars. In the carbon stars, $\lambda 4976$ was tentatively attributed by Wurm ${ }^{11}$ to the convergence of the $\Delta v=0$ sequence of the Swan system of $C_{2}$. A recent unpublished study of these bands in the spectrum of RY Draconis by one of the authors (A. McK.) leads to the conclusion that this identification is almost certainly untenable. Reasons for such a conclusion include the following: (1) The wave length of the band differs by several angstrom units from the convergence wave length calculated from the accurate molecular constants of $C_{2}$. (2) In the spectrum of RY Draconis the $C_{2}$ Swan bands are quite weak, while the unidentified bands, including $\lambda 4975$, are extremely strong. (3) From certain regularities among the unidentified bands, quantities can be derived which are probably the vibrational constants of the molecule responsible for the bands. These do not agree with any known vibrational constants of $C_{2}$. (4) The unidentified bands do not exhibit any isotope effect such as would be expected from a diatomic

${ }^{9}$ Pub. A.S.P., 38, 177, 1926.

${ }^{10}$ Lick Obs. Bull., 13, 123, 1928.

${ }^{11}$ Zs.f. Ap., 13, 179, 1936. 
molecule containing even one, much less both, of its atoms carbon. Therefore, even if the cometary emissions are identical with the above bands (and this is by no means certain), the identity of the molecule responsible for them remains a mystery.

c) The three cometary emissions at $\lambda \lambda$ 5318, 5326, and 5336 may possibly be identified with a new system of $N_{2}$ very recently found by Gaydon. ${ }^{12}$

d) Certain band heads of the red $C N$ system may contribute to cometary features of the visual region.

e) The features included in the table of cometary wave lengths at $\lambda 6059.5$ and $\lambda 6120.9$ are partly due to the $2,4\left(\lambda\right.$ 6059.7) and $1,3\left(\lambda\right.$ 6122.1) bands of the $C_{2}$ Swan system.

f) The cometary emission at $\lambda 5996$ is almost certainly not due to $\mathrm{NO}^{+}$. A strong band with origin at $\lambda 5999$ has been attributed ${ }^{13}$ to $\mathrm{NO}^{+}$, but by comparison with the isoelectronic molecule $C O$ it is certain that this band cannot be a resonance transition of $\mathrm{NO}^{+}$. By analogy with $\mathrm{CO}$ we should expect the absorption required to reach the upper electronic level of the $\lambda 5999$ band of $\mathrm{NO}^{+}$to have to take place in the far ultraviolet, hence excitation by solar radiation to be very inefficient.

g) The cometary emissions at $\lambda 6300$ and $\lambda 6364$ may be occasionally blended with the $[O \mathrm{I}]$ lines of the night-sky radiation.

h) The features at $\lambda \lambda 6053,6097,6148$, and 6200 differ in their appearance from all other features in the visual region. They appear as wide bands, not degraded in either direction, on a faint background. They may be of common origin.

An interesting sidelight in connection with the investigation herein described is that the three authors, while studying the spectrum of Comet Whipple II (1942g) at their respective observatories early in 1943, within a few days of one another quite independently came to the same conclusion regarding the suggested $\mathrm{NH}_{2}$ identifications. Upon learning of this double coincidence through correspondence, they decided to collaborate in the publishing of their combined material.

We wish to express our thanks to Dr. R. S. Mulliken and Dr. G. Herzberg for valuable suggestions and discussions.

12 Nature, 151, 167, 1943.

${ }^{13}$ Duffieux and Grillet, C.R., 202, 937, 1936; Jausseran, Grillet, and Duffieux, C.R., 205, 39, 1937. 